\title{
Hypofractionated stereotactic body radiotherapy in low- and intermediate-risk prostate carcinoma
}

\author{
Hun Jung Kim, MD, Jeong Hoon Phak, MD, Woo Chul Kim, MD \\ Department of Radiation Oncology, Inha University Hospital, Inha University School of Medicine, Incheon, Korea
}

\begin{abstract}
Purpose: Stereotactic body radiotherapy (SBRT) takes advantage of low $\alpha / \beta$ ratio of prostate cancer to deliver a large dose in few fractions. We examined clinical outcomes of SBRT using CyberKnife for the treatment of low- and intermediate-risk prostate cancer. Materials and Methods: This study was based on a retrospective analysis of the 33 patients treated with SBRT using CyberKnife for localized prostate cancer (27.3\% in low-risk and 72.7\% in intermediate-risk). Total dose of $36.25 \mathrm{~Gy}$ in 5 fractions of 7.25 Gy were administered. The acute and late toxicities were recorded using the Radiation Therapy Oncology Group scale. Prostatespecific antigen (PSA) response was monitored.

Results: Thirty-three patients with a median 51 months (range, 6 to 71 months) follow-up were analyzed. There was no biochemical failure. Median PSA nadir was $0.27 \mathrm{ng} / \mathrm{mL}$ at median 33 months and PSA bounce occurred in $30.3 \%(n=10)$ of patients at median at median 10.5 months after SBRT. No grade 3 acute toxicity was noted. The $18.2 \%$ of the patients had acute grade 2 genitourinary (GU) toxicities and 21.2\% had acute grade 2 gastrointestinal (GI) toxicities. After follow-up of 2 months, most complications had returned to baseline. There was no grade 3 late GU and GI toxicity.

Conclusion: Our experience with SBRT using CyberKnife in low- and intermediate-risk prostate cancer demonstrates favorable efficacy and toxicity. Further studies with more patients and longer follow-up duration are required.
\end{abstract}

Keywords: CyberKnife, Prostate cancer, Stereotactic body radiotherapy, Prostate-specific antigen, Radiotherapy, Hypofractionation

\section{Introduction}

The incidence rates of prostate cancer in Korea are relatively lower than those in Western nations but continue to increase [1]. Although intensity-modulated radiation therapy (IMRT) is the standard external beam modality for clinically localized prostate cancer, the use of stereotactic body radiation therapy (SBRT) has recently emerged as a technique to deliver hypofractionated radiation therapy to the prostate as a potent alternative [2-5]. Clinical evidence suggests that the $\alpha /$ $\beta$ ratios of prostate cancer is maybe around $1.5 \mathrm{~Gy}$ and the lower than the surrounding normal tissue $[6,7]$. One phase III study trial suggested that hypofractionation regiment of $62 \mathrm{~Gy}$ in 20 fractions is safe and acute and late complication were equivalent to that of the conventional fractionationated regimen of $80 \mathrm{~Gy}$ in 40 fractions [8].

The CyberKnife is one of the tools for hypofractionated SBRT and real-time image guidance to account for intrafraction prostatic motion. Advanced technique of CyberKnife allows high doses of radiation to be delivered precisely to the target while sparing the surrounding healthy tissue, thus achieving high biochemical control and low toxicity [2-4]. For low and

Received 1 December 2015, Revised 15 December 2015, Accepted 22 December 2015.

Correspondence: Hun Jung Kim, MD, Department of Radiation Oncology, Inha University Hospital, Inha University School of Medicine, 27 Inhang-ro, Jung-gu, Incheon 22332, Korea. Tel: +82-32-890-3070, Fax: +82-32-890-3082, E-mail: cancerovercome@gmail.com

(c) This is an Open Access article distributed under the terms of the Creative Commons Attribution Non-Commercial License (http://creativecommons.org/ licenses/by-nc/4.0/) which permits unrestricted non-commercial use, distribution, and reproduction in any medium, provided the original work is properly cited.

www.e-roj.org 
intermediate risk prostate cancer, recent published literature support use of hypofractionated SBRT using CyberKnife with excellent 5-year biochemical control rates and correspondingly acceptable rates of toxicity $[2,9]$.

We report our experience with CyberKnife SBRT, assessing efficacy and toxicity in treating patients with low and intermediate risk prostate cancer according to new National Comprehensive Cancer Network (NCCN) guidelines.

\section{Materials and Methods}

\section{Patient characteristics}

A prospective protocol-based study for the treatment of localized prostate cancer with CyberKnife Robotic Radiosurgery system began from March 2008 at Inha University Hospital. Since then, 33 patients have been treated. Patients were stratified according to 2.2014 NCCN risk stratification guidelines [10]. Eligible patients had newly diagnosed, biopsyproven low and intermediate risk prostate cancer. The study was approved by the Ethical Committee for Clinical Trials of our institution and the retrospective data was prospectively collected in our institutional database.

\section{SBRT treatment planning and delivery}

Three to four gold fiducial markers were implanted transperineally into the prostate under transrectal ultrasound guidance. On 1 week after fiducial placement, treatment planning CT scans with contrast enhanced were performed at a slice thickness of $1.5 \mathrm{~mm}$ using a multi-slice scanner LightSpeed RT 16 (GE Healthcare, Waukesha, WI, USA). Magnetic resonance imaging scans were obtained with sequences of T1-wighted, gadolinium-enhance. No endorectal coil was used, and the minimum field strength was 1.5 T. Patients had bowel preparation to eliminate rectal contents before treatment-planning scans. All patients underwent computed tomography (CT) simulation in the supine position. $A$ vacuum bag and an ankle holder device was employed as a patient immobilization device. Fused CT and MR images were used for the treatment planning. The prostate, seminal vesicles, rectum, bladder, penile bulb, and bowel were contoured (Fig. 1). The clinical target volume (CTV) included the prostate and proximal seminal vesicles. The planning target volume (PTV) equaled the CTV expanded $3 \mathrm{~mm}$ posteriorly and $5 \mathrm{~mm}$ in all other dimensions. The prescription dose was $36.25 \mathrm{~Gy}$, delivered in 5 fractions, was prescribed to the PTV. Inverse treatment planning was conducted using the MultiPlan Cyberknife treatment planning system ver. 2.2.0 (Accuray
Inc., Sunnyvale, CA, USA). Treatments were performed on 5 consecutive days. The prescription dose covered at least 95\% of the PTV, normalized to the 75\%-85\% isodose line (mean homogeneity index [HI] of 1.26 [range, 1.24 to 1.41]). The $\mathrm{HI}$ describes the uniformity of dose within a treated target volume, and is directly calculated from the prescription isodose line chosen to cover the margin of the tumor: $\mathrm{HI}=$ maximum dose / prescription dose. The rectal dose-volume goals were $<50 \%$ of the rectal volume receiving $50 \%$ of the prescribed dose, $<20 \%$ receiving $80 \%$ of the dose, $<10 \%$ receiving $90 \%$ dose, and $<5 \%$ receiving 100\% of the dose. All patients were treated with the CyberKnife G4 system (Accuray Inc.), composed of a 6-MV linear accelerator mounted on a robotic arm, with two orthogonal kV X-ray imagers that provide realtime stereoscopic image guidance and automatic correction for movements of the prostate throughout treatment with motional tracking system of CyberKnife. Treatments were given over 5 consecutive days. Androgen deprivation therapy was not applied to anyone.

\section{Follow-up and toxicity scoring}

Patients were followed every 3 months during the first year and every 6-12 months thereafter. Prostate-specific antigen (PSA) levels were obtained at each follow-up. Biochemical failure (BCF) was defined as an increase of at least $2 \mathrm{ng} / \mathrm{mL}$ from the nadir PSA according to the Phoenix definition [11] PSA bounce was defined as an absolute increase of $0.2 \mathrm{ng} /$ $\mathrm{mL}$ from the previous PSA level, followed by a subsequent decrease [12]. Toxicity was documented at follow-up visits

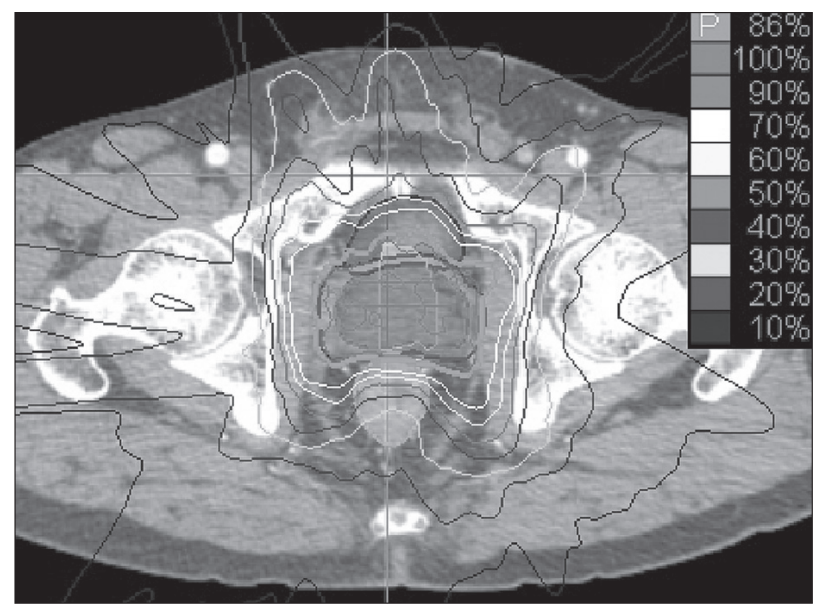

Fig. 1. Stereotactic body radiotherapy using CyberKnife treatment plan of an axial view. The volume represents the prostate (red), planning target volume (blue) and rectum (light blue). The prescription isodose line (86\%) is denoted by the orange line. 
using the Radiation Therapy Oncology Groups. Acute toxicity was defined as occurring within 6 months of completing treatment, and late toxicity as those events occurring later than 6 months. The t-test was performed to compare mean values and ANOVA in continuous variables. BCF-free survival was estimated using the Kaplan-Meier methods. Statistical analysis was performed using IBM SPSS ver. 19.0 (IBM, Armonk, NY, USA).

\section{Results}

\section{PSA response}

The median follow-up duration was 51 months (range, 6 to 71 months). All patients completed the treatment. The median age was 67.2 years (range, 56 to 72 years) (Table 1).

The median pretreatment serum PSA of $7.25 \mathrm{ng} / \mathrm{mL}$ (range, 3.45 to $15.73 \mathrm{ng} / \mathrm{mL}$ ) declined to a median of $0.49 \mathrm{ng} / \mathrm{mL}$ (range, 0.04 to $1.08 \mathrm{ng} / \mathrm{mL}$ ) at 4 years post-treatment (Fig. 2). The decline of PSA was maximal in the first month (median, -2.99 $\mathrm{mg} / \mathrm{mL} / \mathrm{mo}$ ), then decline of PSA was gradually falling off with median values of $-1.07,-0.78,-0.16,-0.13,-0.07,-0.01$, and $>-0.01 \mathrm{ng} / \mathrm{mL} / \mathrm{mo}$ for duration of $3,6,9,12,24,36$, and 48 months after SBRT, respectively. The median PSA nadir was 0.27 $\mathrm{ng} / \mathrm{mL}$ at median 33 months. Benign PSA bounces occurred in 10 patients (30.3\%) with a median PSA bounce of $0.29 \mathrm{ng} /$ $\mathrm{mL}$ (range, 0.22 to $1.36 \mathrm{ng} / \mathrm{mL}$ ) and the median time following treatment to the PSA bounce was 10.5 months (range, 6 to 12

Table 1. Patient and tumor characteristics $(n=33)$

\begin{tabular}{lc}
\hline \multicolumn{1}{c}{ Variable } & Value \\
\hline Age $(\mathrm{yr})$ & $67.2(56-72)$ \\
ECOG performance status & \\
0 & $23(69.7)$ \\
1 & $10(30.3)$ \\
T stage & \\
T1c & $2(6.1)$ \\
T2a-b & $14(42.4)$ \\
T2c & $17(51.5)$ \\
Gleason score & \\
$\leq 6$ & $14(42.4)$ \\
7 & $19(57.6)$ \\
Initial median PSA $(\mathrm{ng} / \mathrm{mL})$ & $7.25(3.45-15.73)$ \\
NCCN risk group & \\
Low & $9(27.3)$ \\
Intermediate & $24(72.7)$ \\
\hline
\end{tabular}

Values are presented as median (range) or number (\%). ECOG, Eastern Cooperative Oncology Group; PSA, prostate-specific antigen; NCCN, National Comprehensive Cancer Network. months). There was no statistically significant difference in age, pretreatment PSA, Gleason score and clinical T-stage with PSA bounce versus no bounce. There was no BCF during the median follow-up of 51 months (range, 6 to 71 months). The 5-year actuarial BCF-free survival rate was 100\%.

\section{Toxicity}

The most common genitourinary (GU) toxicities were urinary frequency and urinary obstructive symptoms. Acute grade $2 \mathrm{GU}$ toxicities were seen in 18.2\% $(n=6)$ (Table 2). Acute GU toxicities were usually resolved within 1-2 month on basic symptomatic therapy. Rectal pain was most prevalent gastrointestinal (GI) toxicity and acute $2 \mathrm{Gl}$ toxicities in $21.2 \%$ $(n=7)$. Acute $\mathrm{Gl}$ toxicity was resolved within 1-2 months with pain medication. No grade 3 or 4 acute GU and GI toxicities were observed. Late grade $2 \mathrm{GU}$ toxicities were observed in $6.1 \%(n=2)$ and grade $2 \mathrm{Gl}$ toxicities in $9.1 \%(n=3)$. Late GU symptoms included nocturia and urinary frequency which were usually controlled by an alpha receptor antagonist. Three patients experienced grade $2 \mathrm{GI}$ toxicities secondary to rectal

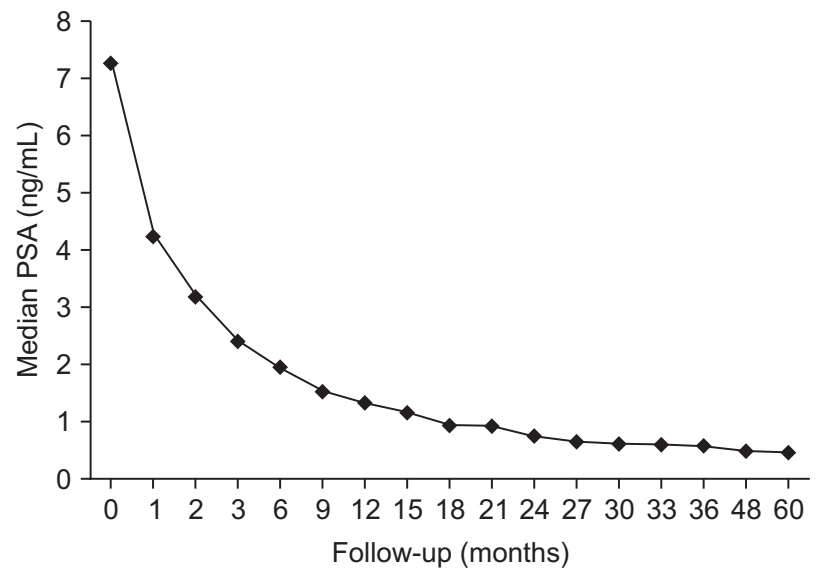

Fig. 2. Prostate-specific antigen (PSA) changes after stereotactic body radiation therapy using CyberKnife.

Table 2. Toxicity (unit, \%)

\begin{tabular}{crccc}
\hline & \multicolumn{5}{c}{ Grade } \\
\cline { 2 - 5 } & I & II & III & IV \\
\hline Acute & & & & \\
GU & 36.3 & 18.2 & - & - \\
GI & 26.4 & 21.2 & - & - \\
Late & & & & \\
GU & 9.1 & 6.1 & - & - \\
GI & 12.1 & 9.1 & - & - \\
\hline
\end{tabular}

GU, genitourinary; GI, gastrointestinal. 
bleeding at 3-6 months after treatment. One patient improved without treatment and two patients improved after laser coagulation. Late toxicity rate was acceptable without severe grade 3 or more GU and GI toxicities.

\section{Discussion and Conclusion}

This report, with follow-up of median 51 months, demonstrates that SBRT using CyberKnife can achieve excellent biochemical control rates while resulting in low levels of bladder and rectal toxicity. Our the 5-year actuarial BCF-free survival rate of $100 \%$ compares favorably with that obtained with surgery and brachytherapy $[13,14]$.

Recent reports show that hypofractionated schedule may provide similar excellent control as other radiation modalities. Arcangeli et al. [15] published a report comparing 80 Gy (2 Gy/fraction) vs. 62 Gy (3.1 Gy/fraction) and showed that the hypofractionated schedule is superior to the conventional fractionation in terms of freedom from BCF rate with equivalent toxicity. This is also confirmed by studies of high dose rate brachytherapy (HDR BT) $[16,17]$. Hoskin et al. [16] showed that HDR BT resulted in an improved BCF-free survival compared to external beam radiotherapy with less acute rectal toxicity and improved quality of life in randomized trial. Demanes et al. [17] reported the 8-year biochemical control of 97\% in low and intermediate risk prostate patients with HDR BT. Our outcomes are consistent with those that have resulted from HDR BT. However, due to its invasive nature and technical difficulties, use of brachytherapy is less common. CyberKnife allows the delivery of large fractions dose such as HDR BT with submillimeter accuracy to the target with excellent sparing of normal tissue. But there is still a matter of debate about the efficacy and toxicity of hypofractionation with CyberKnife.

The rapid decline of PSA level occurred in the first year and PSA fell steadily to achieve very low PSA nadir of median 0.27 $\mathrm{ng} / \mathrm{mL}$. Anwar et al. [18] compared the PSA slope between the hypofractionated SBRT and conventionally fractionated external beam radiation therapy (EBRT) for localized prostate cancer and reported that the PSA slope for SBRT was greater than conventionally fractionated EBRT at 2 and 3 years and PSA nadir was significantly lower for SBRT. Katz et al. [2] demonstrated that PSA decline steadily after treatment and achieve very low mean levels of $0.25 \mathrm{ng} / \mathrm{mL}$ within $4-5$ years. In this study, PSA declined rapidly first year and velocity of decline was gradually falling off with follow up times and declined to median PSA nadir of $0.27 \mathrm{ng} / \mathrm{mL}$ at median 33 months. Several clinical evidence has demonstrated that the $\alpha /$ $\beta$ ratios of prostate cancer is maybe around $1.5 \mathrm{~Gy}[6,7]$. SBRT (5 fraction of $7.25 \mathrm{~Gy}$ ) delivered a BED of $211 \mathrm{~Gy}$, assuming an $\alpha$ / $\beta$ ratio of 1.5 (e.g., BED1.5), compared with a BED1.5 of 154166 Gy with conventionally fractionated EBRT (39-42 fractions of $1.8 \mathrm{~Gy}$ ). Consistent with dose escalation trials which have showed a lower PSA nadir with increased total dose [7], we could expect the SBRT regimen to produce a lower PSA nadir. Lamb et al. [19] showed that the post-radiation nadir PSA is the strongest indicator. Zelefsky et al. [20] demonstrated that nadir PSA values of $\leq 1.5 \mathrm{ng} / \mathrm{mL}$ at 2 years after radiation therapy for prostate cancer predict for long-term distant metastases and cause-specific mortality. We regard the low nadir of $0.27 \mathrm{ng} / \mathrm{mL}$ in our report as indicative of a favorable outcome despite the limited follow-up.

In this study, PSA bounce was seen in $30.3 \%$ of patients after SBRT. McBride et al. [5] found that the mean age of those who experienced a bounce was significantly younger than those who did not. Vu et al. [21] reported that younger age was the only factor that predicted PSA bounce following SBRT for prostate cancer. Park et al. [22] showed that only pretreatment PSA level was associated with increased risk of PSA bounce. However, prognostic factors such as age, pretreatment PSA, Gleason score and clinical T-stage were not associated with PSA bounce in our study.

Toxicity following SBRT was similar to that following EBRT or brachytherapy. Zelefsky et al. [20] reported result on late toxicity using 81 Gy dose with IMRT in conventional fractionation. The 8-year actuarial likelihood of grade $2 \mathrm{Gl}$ toxicity was $1.6 \%$ and $0.1 \%$ of patients experienced grade 3 rectal toxicity. The 8-year likelihood of late grade 2 and $3 \mathrm{GU}$ toxicities were 9\% and 3\%, respectively. Our current study shows the similar proportion of toxicity.

Our study should be examined in the context of study design. Our study is limited by retrospective nature of the analysis and the small number of patients. There were no strict protocols for the clinical decision-making process. Future studies should employ more comprehensive instruments to assess the effect of prostate SBRT.

The finding of our study was very encouraging. The biochemical disease control is comparable to other available therapies, with equal to or better toxicity profiles. We look forward to future multicenter studies that will examine outcomes with this treatment approach.

\section{Conflict of Interest}

No potential conflict of interest relevant to this article was 
reported.

\section{Acknowledgments}

This work was supported by Inha University Research Grant.

\section{References}

1. Jung KW, Won YJ, Kong HJ, et al. Cancer statistics in Korea: incidence, mortality, survival, and prevalence in 2012. Cancer Res Treat 2015;47:127-41.

2. Katz AJ, Santoro M, Diblasio F, Ashley R. Stereotactic body radiotherapy for localized prostate cancer: disease control and quality of life at 6 years. Radiat Oncol 2013;8:118.

3. Chen LN, Suy $S$, Uhm S, et al. Stereotactic body radiation therapy (SBRT) for clinically localized prostate cancer: the Georgetown University experience. Radiat Oncol 2013;8:58.

4. King CR, Brooks JD, Gill H, Presti JC Jr. Long-term outcomes from a prospective trial of stereotactic body radiotherapy for low-risk prostate cancer. Int J Radiat Oncol Biol Phys 2012;82: 877-82.

5. McBride SM, Wong DS, Dombrowski JJ, et al. Hypofractionated stereotactic body radiotherapy in low-risk prostate adenocarcinoma: preliminary results of a multi-institutional phase 1 feasibility trial. Cancer 2012;118:3681-90.

6. Fowler JF, Ritter MA, Chappell RJ, Brenner DJ. What hypofractionated protocols should be tested for prostate cancer? Int J Radiat Oncol Biol Phys 2003;56:1093-104.

7. Brenner DJ, Hall EJ. Fractionation and protraction for radiotherapy of prostate carcinoma. Int J Radiat Oncol Biol Phys 1999:43:1095-101.

8. Arcangeli G, Fowler J, Gomellini S, et al. Acute and late toxicity in a randomized trial of conventional versus hypofractionated three-dimensional conformal radiotherapy for prostate cancer. Int J Radiat Oncol Biol Phys 2011;79:1013-21.

9. Freeman DE, King CR. Stereotactic body radiotherapy for lowrisk prostate cancer: five-year outcomes. Radiat Oncol 2011;6:3.

10. Mohler JL, Kantoff PW, Armstrong AJ, et al. Prostate cancer, version 2.2014. J Natl Compr Canc Netw 2014;12:686-718.

11. Abramowitz MC, Li T, Buyyounouski MK, et al. The Phoenix definition of biochemical failure predicts for overall survival in patients with prostate cancer. Cancer 2008;112:55-60.

12. Patel $C$, Elshaikh MA, Angermeier $K$, et al. PSA bounce predicts early success in patients with permanent iodine-125 prostate implant. Urology 2004;63:110-3.

13. D'Amico AV, Whittington $R$, Malkowicz SB, et al. Biochemical outcome after radical prostatectomy or external beam radiation therapy for patients with clinically localized prostate carcinoma in the prostate specific antigen era. Cancer 2002;95:281-6.

14. Kupelian PA, Potters L, Khuntia D, et al. Radical prostatectomy, external beam radiotherapy $<72 \mathrm{~Gy}$, external beam radiotherapy $>$ or $=72 \mathrm{~Gy}$, permanent seed implantation, or combined seeds/external beam radiotherapy for stage T1-T2 prostate cancer. Int J Radiat Oncol Biol Phys 2004;58:25-33.

15. Arcangeli G, Saracino B, Gomellini S, et al. A prospective phase III randomized trial of hypofractionation versus conventional fractionation in patients with high-risk prostate cancer. Int J Radiat Oncol Biol Phys 2010;78:11-8.

16. Hoskin PJ, Motohashi K, Bownes P, Bryant L, Ostler P. High dose rate brachytherapy in combination with external beam radiotherapy in the radical treatment of prostate cancer: initial results of a randomised phase three trial. Radiother Oncol 2007;84:114-20.

17. Demanes DJ, Martinez AA, Ghilezan M, et al. High-dose-rate monotherapy: safe and effective brachytherapy for patients with localized prostate cancer. Int J Radiat Oncol Biol Phys 2011;81:1286-92.

18. Anwar M, Weinberg V, Chang AJ, Hsu IC, Roach M 3rd, Gottschalk A. Hypofractionated SBRT versus conventionally fractionated EBRT for prostate cancer: comparison of PSA slope and nadir. Radiat Oncol 2014;9:42.

19. Lamb DS, Denham JW, Joseph $D$, et al. A comparison of the prognostic value of early PSA test-based variables following external beam radiotherapy, with or without preceding androgen deprivation: analysis of data from the TROG 96.01 randomized trial. Int J Radiat Oncol Biol Phys 2011;79:385-91.

20. Zelefsky MJ, Chan H, Hunt M, Yamada Y, Shippy AM, Amols $\mathrm{H}$. Long-term outcome of high dose intensity modulated radiation therapy for patients with clinically localized prostate cancer. J Urol 2006;176(4 Pt 1):1415-9.

21. Vu CC, Haas JA, Katz AE, Witten MR. Prostate-specific antigen bounce following stereotactic body radiation therapy for prostate cancer. Front Oncol 2014;4:8.

22. Park YH, Choi IY, Yoon SC, et al. Prostate-specific antigen kinetics after primary stereotactic body radiation therapy using CyberKnife for localized prostate cancer. Prostate Int 2015;3:6-9. 